\title{
Neuroevolution: from architectures to learning
}

\author{
Dario Floreano · Peter Dürr · Claudio Mattiussi
}

Received: 5 October 2007 / Accepted: 17 October 2007/Published online: 10 January 2008

(C) Springer-Verlag 2008

\begin{abstract}
Artificial neural networks (ANNs) are applied to many real-world problems, ranging from pattern classification to robot control. In order to design a neural network for a particular task, the choice of an architecture (including the choice of a neuron model), and the choice of a learning algorithm have to be addressed. Evolutionary search methods can provide an automatic solution to these problems. New insights in both neuroscience and evolutionary biology have led to the development of increasingly powerful neuroevolution techniques over the last decade. This paper gives an overview of the most prominent methods for evolving ANNs with a special focus on recent advances in the synthesis of learning architectures.
\end{abstract}

Keywords Neural networks $\cdot$ Evolution $\cdot$ Learning

\section{Introduction}

Over the last 50 years, researchers from a variety of fields have used models of biological neural networks not only to better understand the workings of biological nervous systems, but also to devise powerful tools for engineering applications.

\footnotetext{
D. Floreano $\cdot$ P. Dürr $(\square) \cdot$ C. Mattiussi

Ecole Polytechnique Fédérale de Lausanne Laboratory

of Intelligent Systems, Station 11, 1015 Lausanne, Switzerland

e-mail: peter.duerr@epfl.ch

URL: http://lis.epfl.ch

D. Floreano

e-mail: dario.floreano@epfl.ch

C. Mattiussi

e-mail: claudio.mattiussi@epfl.ch
}

Artificial neural networks (ANNs) are computational models implemented in software or specialized hardware devices that attempt to capture the behavioral and adaptive features of biological nervous systems. They are typically composed of several interconnected processing units, or 'neurons' (see Fig. 1) which can have a number of inputs and outputs. In mathematical terms, an ANN can be seen as a directed graph where each node implements a neuron model. In the simplest case, the neuron model is just a weighted sum of the incoming signals transformed by a (typically nonlinear) static transfer function. More sophisticated neuron models involve discrete-time or continuoustime dynamics (see Sect. 3). The connection strengths associated with the edges of the graph connecting two neurons are referred to as synaptic weights, the neurons with connections to the external environment are often called input or output neurons, respectively. The number and type of neurons and the set of possible interconnections between them define the architecture or topology of the neural network.

In order to solve computational or engineering problems with neural networks, learning algorithms are used to find suitable network parameters. Evolutionary algorithms provide an interesting alternative, or complement, to the commonly used learning algorithms, such as back-propagation $[68,69]$. Evolutionary algorithms are a class of population-based, stochastic search methods inspired by the principles of Darwinian evolution. Instead of using a conventional learning algorithm, the characteristics of neural networks can be encoded in artificial genomes and evolved according to a performance criterion. The advantages of using an evolutionary algorithm instead of another learning method are that several defining features of the neural network can be genetically encoded and co-evolved at the same time and that the definition of a performance 


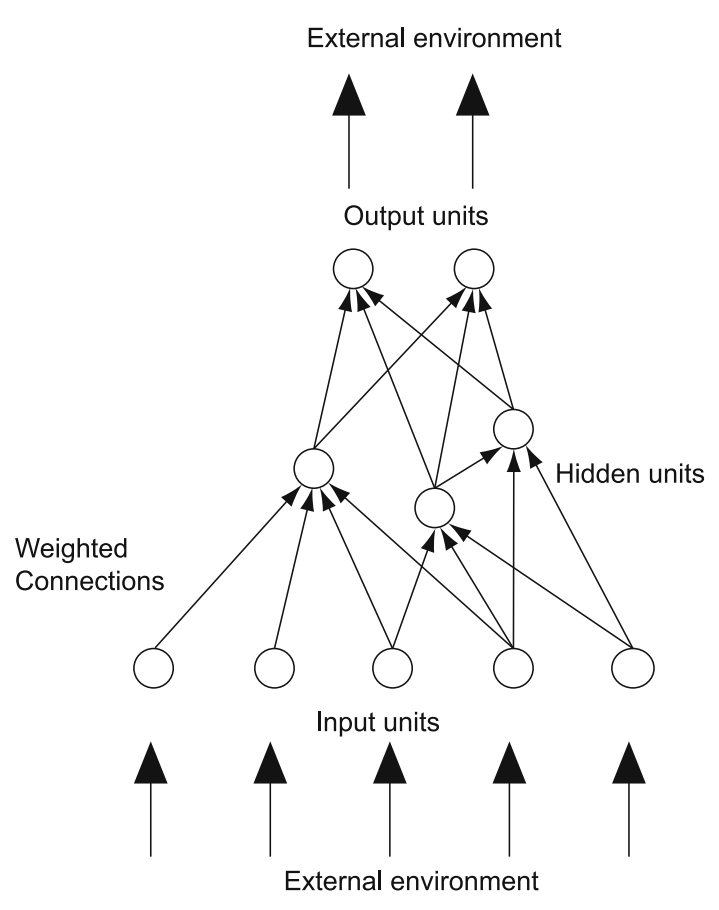

Fig. 1 A generic neural network architecture. It consists of Input units and Output units which are connected to the external environment and Hidden units which connect to other neurons but are not directly connected to the environment

criterion is more flexible than the definition of an energy or error function. Furthermore, evolution can be coupled with a learning algorithm such as Hebbian learning [32], or even used to generate new learning algorithms. In the following we give an overview of the recent methods for evolving ANNs. Since to our knowledge there is no systematic large-scale comparison of the different approaches we focus on the most-widely used canonical methods and some of the most promising recent developments. We first review approaches that evolve the network architecture and its parameters. Then we give a short overview of the most prominent dynamic neuron models used in neuroevolution experiments. We then move on to describe different methods for combining evolution and learning, including the evolutionary synthesis of novel learning algorithms. Finally we point to recent work aiming at evolving architectures capable of controlling learning events.

\section{Evolution of neural architectures}

The evolutionary synthesis of a neural network leads to several design choices. Besides the choice of an appropriate fitness function and the setting of appropriate evolutionary parameters, e.g., population size or mutation rates, the key problem is the choice of suitable genetic representations that recombination and mutation operators can manipulate to discover high-fitness networks with high probability. We can classify current representations into three classes: direct, developmental and implicit. Although all three representations can be used to evolve both the network topology and its parameters, developmental and implicit representations offer more flexibility for evolving neural topologies whereas direct representations have been used mainly for evolving the parameter values of fixed-size networks.

\subsection{Direct Representations}

In a direct representation, there is a one-to-one mapping between the parameter values of the neural network and the genes that compose the genetic string. In the simplest case, synaptic weights and other parameters (e.g., bias weights or time-constants) of a fixed network topology are directly encoded in the genotype either as a string of real values or as a string of characters, which are then interpreted as realvalues with a given precision. This can be done by interpreting the string of characters as a binary or Gray-coded number. However, this strategy requires the knowledge of the suitable intervals and precision of the encoded parameters. In order to make the mapping more adaptive, Schraudolph and Belew [73] suggested a dynamic encoding, where the bits allocated for each weight are used to encode the most significant part of the binary representation until the population has converged to a satisfactory solution. At that point, those same bits are used to encode the less significant part of the binary representation in order to narrow the search and refine the performance of the evolutionary network. Another possibility is to use a selfadaptive encoding such as the Center of Mass Encoding suggested by Mattiussi et al. [45], where the characters of the string are interpreted as a system of particles whose center of mass determines the encoded value. The advantage of this method is that it automatically adapts the granularity of the representation to the requirements of the task.

Recent benchmark experiments with Evolution Strategies [62], which use a floating-point representation of the synaptic weights, have reported excellent performance with direct encoding of a small, fixed architecture [38]. In these experiments, Igel used an evolution strategy called covariance matrix adaptation (CMA-ES). CMA-ES is an evolutionary algorithm that generates new candidate solutions by sampling from a multivariate normal distribution over the search space and changes this mutation distribution by adapting its covariance matrix [30].

In a classic study, Montana and Davis [51] compared the performance of synaptic weight evolution with a discrete direct representation with that of the back-propagation 
algorithm on a problem of sonar data classification. The results indicate that evolution finds much better networks and in significantly less computational cycles than backpropagation of error (the evaluation of one evolutionary individual on the data set is equivalent to one set of training cycles on the data set). These results have been confirmed for a different classification task by other authors [88]. Evolved neural networks with direct encoding have also been applied to a variety of other problems such as game playing [13], data classification [12] or the control of robot swarms [85].

It has been argued $[72,61]$ that evolving neural networks may not be trivial because the population may include individuals with competing conventions (see Fig. 2). This refers to the situation where very different genotypes (conventions) correspond to neural networks with similar behavior. For example, two networks with inverted hidden nodes may have very different genotypes, but will produce exactly the same behavior. Since the two genotypes correspond to quite different areas of the genetic space, crossover among competing conventions may produce offspring with duplicated structures and low fitness. Another problem that evolutionary algorithms for ANNs face is premature convergence. Difficult fitness landscapes with local optima can cause a rapid decrease of the population diversity and thus render the search inefficient.

To overcome these problems, Moriarty and Miikkulainen [52] suggested to evolve individual neurons to cooperate in networks. This has the advantage that population diversity is automatically maintained and competing conventions can be avoided. The authors developed an algorithm called symbiotic, adaptive neuro-evolution (SANE). They encoded the neurons in binary chromosomes which contained a series of connection definitions (see Fig. 3). The neurons connected only to the input and the output layer. During the evaluation stage, random neurons were selected to form a neural network of fixed size. The fitness of each neuron was defined as the average fitness of all the networks it had participated in. Gomez and Miikkulainen [27] extended this approach by segregating the neurons in subpopulations with a method they called enforced subpopulations (ESP). SANE could not evolve recurrent neural networks as the neurons were selected randomly from the population and thus could not rely on being combined with similar neurons in different trials. The subpopulations of ESP resolved this problem and additionally allowed for a higher amount of specialization through constrained mating. ESP has been applied to various benchmark tasks, for example several different pole balancing setups, however, more recent methods such as Neuro-Evolution of Augmenting Topologies (see below) and Analog Genetic Encoding (see Sect. 2.3) outperformed ESP on these benchmarks [16, 79].

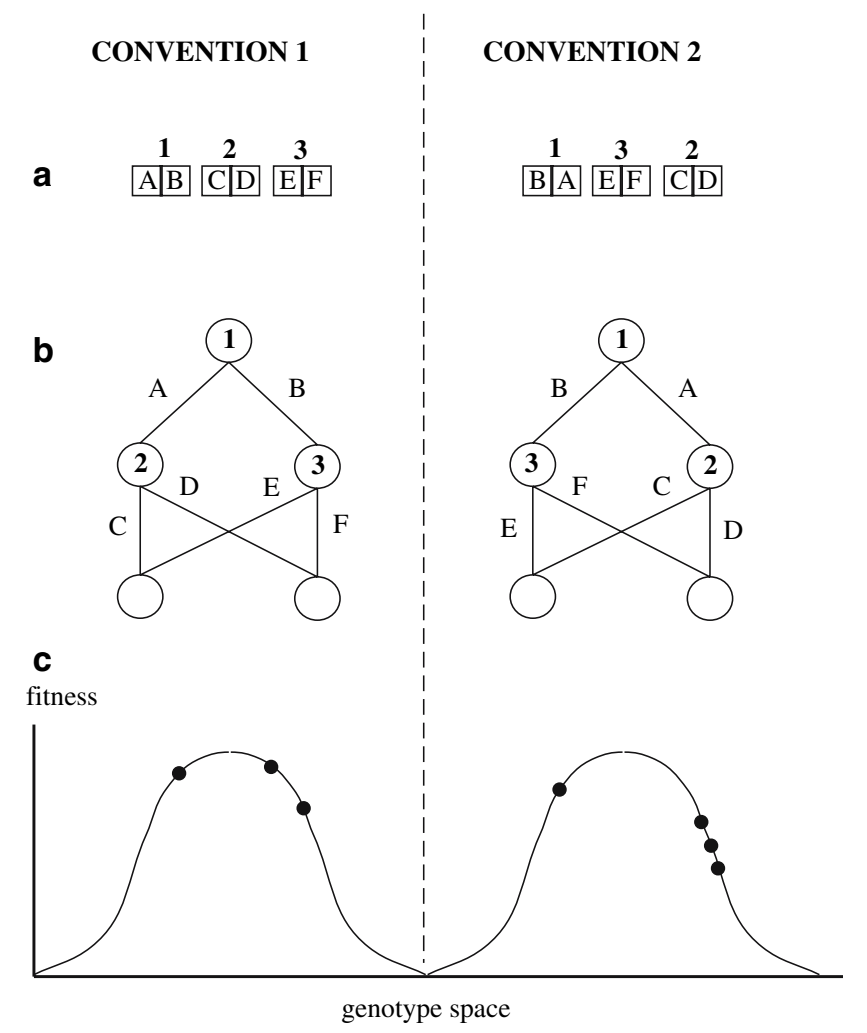

Fig. 2 Competing conventions. Two different genotypes (a) may encode networks that are behaviorally equivalent, but have inverted hidden units (b). The two genotypes define two separate hills on the fitness landscape (c) and thus may make evolutionary search more difficult. Adapted from Schaffer et al. [72]

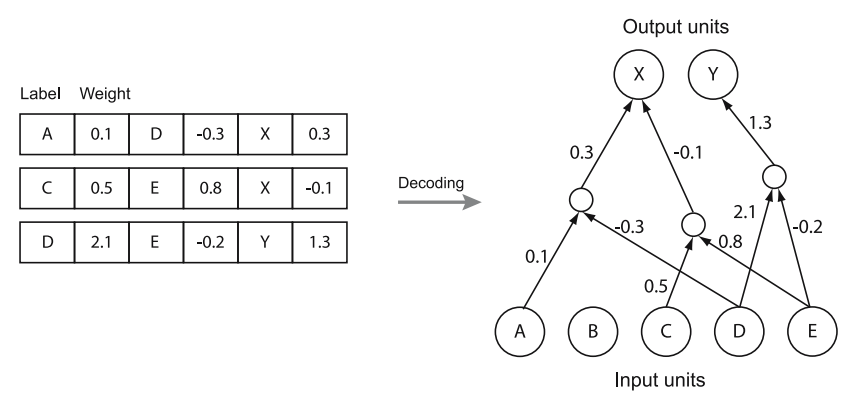

Fig. 3 The symbiotic, adaptive neuroevolution (SANE) encoding. Each individual of the population encodes a neuron with a series of connection definitions. The value of the label defines to which input or output the neuron connects. The weight field encodes the respective connection weight. The final neural network is formed by combining randomly chosen neurons from the population. Redrawn from Moriarty and Miikkulainen [52]

The topology of a neural network can significantly affect its ability to solve a problem. As mentioned above, direct encoding is typically applied to fixed network topologies, however, it can also be used to evolve the architecture of an ANN. One of the main challenges of evolving the topology along with the weights and parameters of a neural network is that changes in the topology usually lead to decreased 
fitness even if they have the potential to further increase the fitness later in evolution [79].

Neuro-evolution of augmenting topologies (NEAT) is a method for genetically encoding and evolving the architecture and the weights of neural networks [79]. The approach makes use of genetic operators that can introduce new genes and disable old ones. NEAT was designed to avoid the problem of competing conventions, allowing meaningful crossover between individuals with different genetic length, produce networks of increasing complexity starting from simple ones, and protect topological innovations that may initially display lower fitness but later develop into powerful solutions.

The main insight of NEAT is that genes sharing the same evolutionary origin are more likely to encode a similar function. In order to keep a genetic historical record, whenever a new gene is created, it is assigned a marker that corresponds to its chronological order of appearance in the evolving population (see Fig. 4). When genes are reproduced and transmitted to offspring, they retain their original markers. The marker number is used to find homologous genes that correspond to alignment points between genotypes of different length, to prevent crossover on competing conventions, and to detect the genetic similarity of individuals in order to create subpopulations of similar individuals. Selective reproduction operates on individuals within the same sub-population and the fitness of an individual is divided by a number proportional to the number of individuals that are genetically similar.

This last feature is useful for preventing the competition for reproduction between old individuals, which have a relatively high fitness, and newer individuals with topological innovations (genes with high innovation numbers), which may display relatively low fitness. Since the two types of individuals will be genetically different, they will

\begin{tabular}{|c|c|c|c|}
\hline \multicolumn{4}{|c|}{ NEAT genome } \\
\hline \multicolumn{4}{|l|}{ Node genes } \\
\hline $\begin{array}{l}\text { Node } 1 \\
\text { Sensor }\end{array}$ & $\begin{array}{l}\text { Node } 2 \\
\text { Sensor }\end{array}$ & $\begin{array}{l}\text { Node } 3 \\
\text { Hidden }\end{array}$ & $\begin{array}{l}\text { Node } 4 \\
\text { Output }\end{array}$ \\
\hline \multicolumn{4}{|c|}{ Connection genes } \\
\hline $\begin{array}{l}\text { In } 3 \\
\text { Out } 4 \\
\text { Weight } 0.7 \\
\text { Enabled } \\
\text { Innov } 1\end{array}$ & \multicolumn{2}{|c|}{$\begin{array}{l}\text { In } 1 \\
\text { Out } 4 \\
\text { Weight } 0.2 \\
\text { Enabled } \\
\text { Innov } 5\end{array}$} & $\begin{array}{l}\text { In } 2 \\
\text { out } 4 \\
\text { Weight }-1 \\
\text { DISABLED } \\
\text { Innov } 3\end{array}$ \\
\hline $\begin{array}{l}\text { In } 2 \\
\text { Out } 3 \\
\text { Weight } 0.3 \\
\text { Enabled } \\
\text { Innov } 4\end{array}$ & \multicolumn{2}{|c|}{$\begin{array}{l}\text { In } 4 \\
\text { Out } 3 \\
\text { Weight }-1 \\
\text { Enabled } \\
\text { Innov } 9\end{array}$} & $\begin{array}{l}\text { In } 1 \\
\text { Out } 3 \\
\text { Weight } 0.5 \\
\text { Enabled } \\
\text { Innov } 10\end{array}$ \\
\hline
\end{tabular}

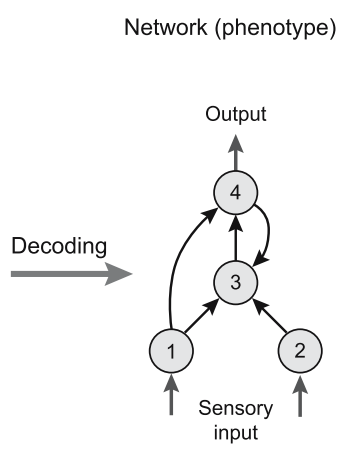

Fig. 4 Genetic encoding of a network topology within NEAT. Genetic operators can insert new genes or disable old genes. When a new gene is inserted, it receives an innovation number that marks its inception. Redrawn from Stanley and Miikkulainen [79] compete separately for reproduction. NEAT starts with an initial population where genotypes correspond to neural networks of minimal size. The genetic operators can modify the genotypes by inserting new genes that correspond to larger networks. If those larger networks provide a competitive advantage, they are retained and compete with networks of different size.

Neuro-evolution of augmenting topologies (NEAT) has been applied to many problems such as pole balancing [79], robot control [80], computer games [66, 82] or an automobile crash warning system [81].

Direct representations have been used with excellent results for networks of relatively small size. However, they may not work well for larger networks because the length of the genetic string and its evolvability do not always scale well with network size.

\subsection{Developmental representations}

In order to evolve large networks, some researchers suggested to genetically encode the specification of a developmental process which in turn constructs the neural network. In a seminal paper, [40] suggested a developmental encoding based on a set of rewriting rules encoded in the genotype (see Fig. 5). He employed a genome divided into blocks of five elements. Each block of five is interpreted as a rewriting rule that determines how the first symbol is developed into a matrix containing the other four symbols of the block. There are two types of symbols: terminals and non-terminals. A terminal symbol develops into a predetermined $2 \times 2$ matrix of $0 \mathrm{~s}$ and $1 \mathrm{~s}$. A nonterminal symbol develops into a $2 \times 2$ matrix of symbols. The first block of the genotype builds the initial $2 \times 2$ matrix of symbols, each of which recursively develops using the rules encoded in the genotype until a matrix of 0 s and 1s is built. This matrix represents the architecture and connection pattern of the network. Kitano's experiments indicated that such a developmental encoding led to better results than a direct encoding when used to evolve the connectivity pattern of a neural network that was subsequently trained with back-propagation. However, Siddiqi and Lucas [76] showed that the inferior performance of the direct representation in Kitano's work did not result from the difference in the network representations but from different initial populations.

Among others, (see Yao [91] for more examples), Gruau [28] proposed a genetic encoding scheme for neural networks based on a cellular duplication and differentiation process, i.e., a cellular encoding (CE). The genotype-tophenotype mapping starts with a single cell that undergoes a number of duplication and transformation processes resulting in a complete neural network. In this scheme the 
Fig. 5 Kitano's grammar encoding. The genome is divided into blocks of five elements. Each block defines a rewriting rule. Together with a set of fixed rules, this allows the recursive development of a matrix which defines the connectivity of a neural network. Redrawn from Kitano [40]
Genome

SABCDBa a a e Da a a b...
Evolvable rules
$\mathrm{S} \longrightarrow \mathrm{AB}$
$\mathrm{A} \longrightarrow \begin{aligned} & \mathrm{CP} \\ & \mathrm{aC}\end{aligned}$
$\mathrm{B} \longrightarrow \mathrm{a}$ a
$\mathrm{C} \longrightarrow \mathrm{aa}$
$\mathrm{D} \longrightarrow \mathrm{a} \mathrm{a}$
Fixed rules

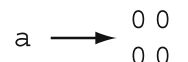
$\mathrm{b} \longrightarrow \begin{aligned} & 0 \\ & 0\end{aligned}$
$\mathrm{C} \longrightarrow \begin{array}{ll}1 & 0 \\ 0 & 0\end{array}$
$\mathrm{e} \longrightarrow \begin{aligned} & 01 \\ & 01\end{aligned}$
$\mathrm{p} \longrightarrow \begin{aligned} & 1 \\ & 1 \\ & 1\end{aligned}$

Connectivity definition for a feed forward network

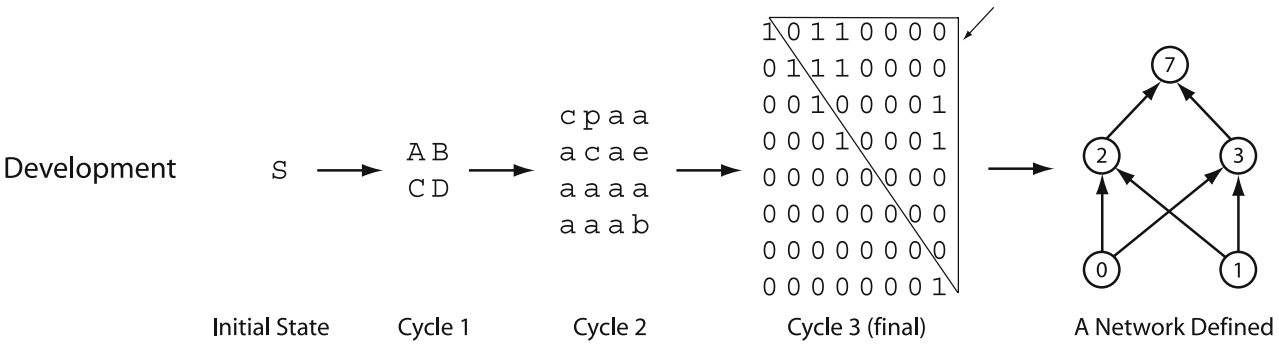

genotype is a collection of rules governing the process of cell divisions (a single cell is replaced by two "daughter" cells) and transformations (new connections can be added and the weights of the connections departing from a cell can be modified).

In Gruau's model, connection links are established during the cellular duplication process. For this reason, there are different forms of duplication, each determining the way in which the connections of the mother cells are inherited by the daughter cells. Additional operations include the possibility to add or remove connections and to modify the connection weights. The instructions contained in the genotype are represented with a binary tree structure and evolved using genetic programming [4]. During the genotype-to-phenotype mapping process, the genotype tree is scanned starting from the top node of the tree and then following each ramification. Inspired by Koza's work on automatic discovery of reusable programs [42], Gruau also considered the case of genotypes formed by many trees where the terminal nodes of a tree may point to other trees. This mechanism allows the genotype-to-phenotype process to produce repeated phenotypical structures (e.g., repeated neural sub-networks) by re-using the same genetic information. Trees that are pointed to more than once will be executed more often. This encoding method has two advantages: (a) compact genotypes can produce complex phenotypical networks, and (b) evolution may exploit phenotypes where repeated substructures are encoded in a single part of the genotype. Since the identification of substructures that are read more than once is an emergent result of the evolutionary process, Gruau called this cellular encoding automatic definition of neural subnetworks (ADNS). Cellular encodings have been applied to different control problems such as pole balancing [29] or the control of a hexapod robot [28].

In the cellular encoding, development occurs instantaneously before evaluating the fully formed phenotype. Nolfi et al. [57] used a growing encoding scheme to evolve the architecture and the connection weights of neural networks controlling a Khepera robot, where the growth of the neural network occurred during the lifetime of the robot while its fitness was evaluated. These controllers were composed of a collection of artificial neurons distributed over a two-dimensional space with growing and branching 'axons' (see Fig. 6). The genotype specified instructions that controlled the axonal growth and branching process of

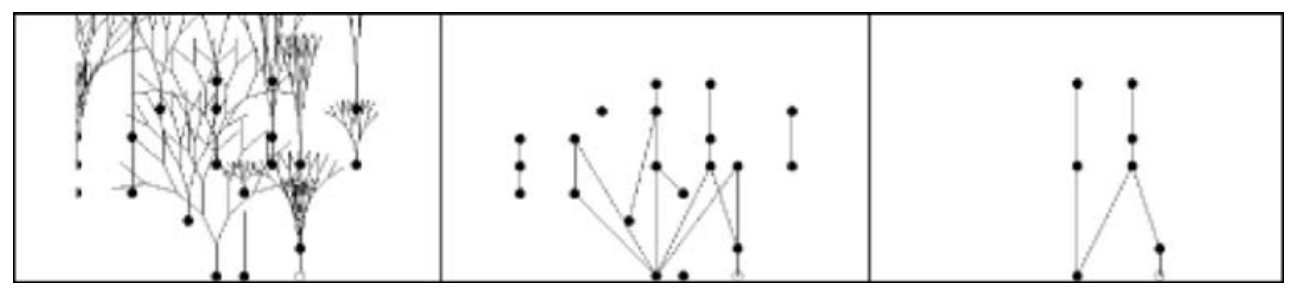

Fig. 6 Growth of axonal connections in the 2D brain space, pruning of axons that do not connect to other neurons, and pruning of all neurons that are not connected to input, output, or other parts of the network. From Nolfi et al. [57] 
Fig. 7 a In biological gene networks, the link between genes is realized by molecules that are synthesized from the coding region of one gene and interact with the regulatory region of another gene. b Analog genetic encoding abstracts this mechanism with an interaction map that transforms the coding and regulatory regions into a numerical value that represents the strength of the link. From Mattiussi et al. [46]

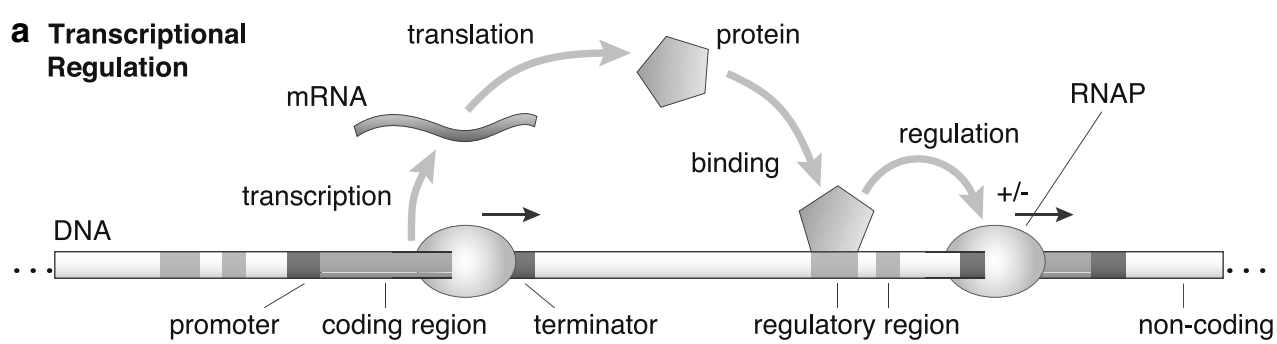

b The AGE Abstraction

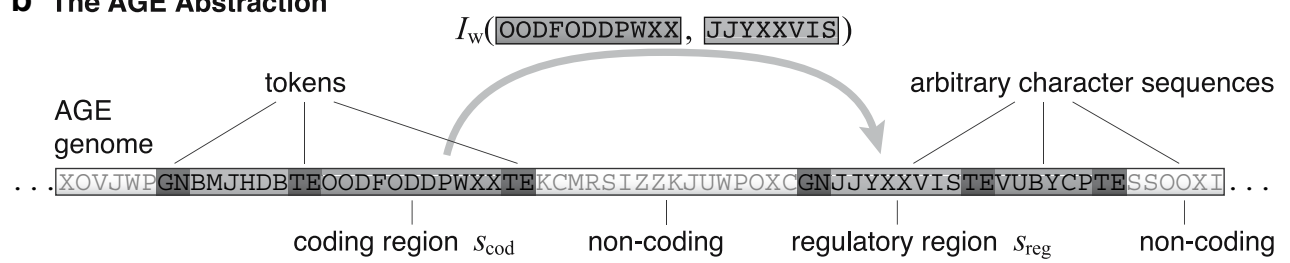

a set of neurons. When the axon growing from one neuron reached another neuron, a connection between the two neurons was established. Axons grew and branched only if the neurons displayed an activation variability above a genetically specified threshold. Axons that did not connect to other neurons and neurons that remained unconnected were pruned.

This activity-dependent growth was based on the idea that the sensory information coming from the environment plays a critical role in the maturation of the connectivity of the biological nervous system. Indeed, it has been shown that the maturation process is affected by the activity patterns of single neurons $[59,60]$. The developmental process of these individuals was therefore determined both by genetic and by environmental factors.

Husbands et al. [36] proposed a similar method where the connections grew according to a set of differential equations. The genotype encoded the properties of each neuron (the type of neuron, the relative position with respect to the neuron created previously, the initial direction of growth of the dendrites and the parameters of the equations governing the growth process). During the genotype-tophenotype process, the genetic string was scanned from left to right until a particular marker was found. When a special marker indicating the beginning of the description of a neuron was encountered, the following bits were read and interpreted as parameters for a new neuron. The presence of an additional marker, however, could indicate that the parameters of the current neuron were specified in a previous portion of the string. This mechanism could potentially allow the emergence of phenotypes with repeated structures formed by re-expression of the same genetic instructions, similarly to the approach described by Gruau.

Despite the ability of developmental approaches to generate large networks from compact genetic representations, it is not clear to what extent the network size contributes to solve a problem. Developmental representations tend to generate regular and modular architectures, which can be very suitable for problems that require them, such as the control of a multi-legged robot. However, these methods require additional mechanisms to cope with asymmetric architectures and fine-tuning of specific parameters.

\subsection{Implicit encoding}

A recent approach to representing neural networks is inspired from the mechanisms of biological gene regulatory networks (GRNs). The so-called implicit encoding is frequently used as a representation for GRN models [10] and has also been considered as a representation for other types of networks, such as neural networks.

In biological gene networks, the interaction between the genes is not explicitly encoded in the genome, but follows implicitly from the physical and chemical environment in which the genome is immersed. The activation of a gene results from interactions of proteins in the environment of the gene with the so-called regulatory regions (see Fig. 7a). These are sequences of nucleotides to which the proteins can bind to promote or hinder the working of specialized molecular machinery that is in charge of expressing the gene. The expression of the gene corresponds to the synthesis of proteins specified in another sequence of genetic characters, the so-called coding region of the gene. The start and end of the coding region of a gene are marked by special motifs (i.e., nucleotide patters), called promoter and terminator regions. The proteins produced by a gene can in turn interact with the regulatory regions of other genes and influence their expression. This process can be interpreted as a network of interactions between the different genes. 

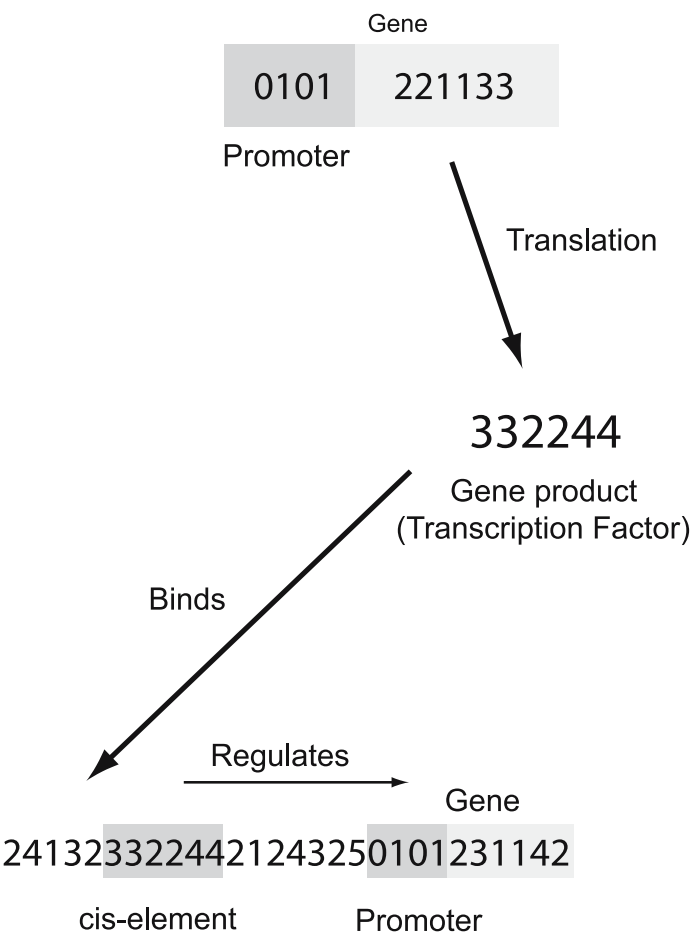

Fig. 8 A simple model of gene expression and regulation in artificial genomes. The genes which are marked by promoter regions in the genome, are translated into transcription factors. These transcription factors are then used to regulate the expression of the following genes. Redrawn from Reil [64]

Reil $[63,64]$ studied the structural properties of such gene networks with a simple computational model. He used a sequence of characters from a finite alphabet as a genome. The presence of a gene in the genome is signaled by the presence of a promoter, that is, a predefined sequence of fixed length. A gene is constituted by a fixed, predefined number of characters following a promoter. In Reil [63], an activated gene produces another sequence of characters which is a transliterated version of the gene. The new sequence is interpreted as a regulatory protein. If a regulatory protein matches exactly a sequence of characters in the genome, it regulates the expression of the gene that immediately follows the matched genome sequence (see Fig. 8). The regulation can be positive or negative, and is of the on-off type, with repression prevailing over activation. Geard and Wiles [25] refined this model by complexifying the map that transforms a gene into a regulator protein, adding a further level of regulation which mimics the action of small RNA regulation in real genomes and defining regulation in terms of a weighted sum of regulator proteins effects. The simulation of such artificial GRNs produced behaviors featuring many properties observed in real GRNs. Although these early computational GRN models were not directly aimed at the exploitation of the evolutionary properties of such a system, they inspired other researchers such as Bongard
[10] to use GRN models to evolve autonomous agents. Bongard used a GRN model that relied on the diffusion of chemicals, so-called transcription factors, which could either directly influence the phenotype of the agent or regulate the expression of genes. The results of his computational experiments showed that his simple model allowed for the evolution of complex morphologies capable of simple locomotion behaviors and indicated that the evolution of modular GRNs was beneficial for the success of the agents.

This type of abstraction of the biochemical process of gene regulation can be extended to a genetic representation for any kind of analog network. ${ }^{1}$ Analog genetic encoding (AGE) is such a representation which has been applied to the evolution of ANNs [15, 44, 46]. AGE is based on a discrete genome composed of sequences of characters from a finite alphabet (e.g., the ASCII uppercase alphabet) which is decoded into a network of interconnected devices (see Fig. 7b). In the case of an artificial neural network, the different types of neurons of the network are encoded by sequences of genome which are separated by predefined delimiter sequences. These artificial coding and regulatory regions play a role analogous to the corresponding sequences in biological genomes.

The biochemical process which determines the interaction between the proteins encoded by one gene and the regulatory region of another gene is abstracted by the socalled interaction map. The interaction map is a mathematical function that takes two sequences of characters as argument and outputs a numerical value representing the synaptic weight between two neurons. The neural network can be decoded from the genome by scanning it for the sequences of characters which represent a neuron. Subsequently, the interaction map can be used to compute the interaction strengths between these neurons (see Fig. 9). The size and topology of the decoded network hence depends on the number of neurons encoded in the genome and the interaction strengths between them (which can be zero, thus leading to no connection between the corresponding neurons).

In AGE, the sequences that define the interaction between devices can have variable length, and the interaction map that is used to establish the connection between the neurons is defined so as to apply to sequences of arbitrary length. The assemblies of sequences of characters that represent a neuron can be located anywhere in the genome and can be spaced by stretches of non-coding genetic characters. In this way the structure of the genome

\footnotetext{
${ }^{1}$ Analog networks are collections of dynamical devices interconnected by links of varying strength. For example, genetic regulatory networks, metabolic networks, neural networks, or electronic circuits can be seen as analog networks.
} 
Fig. 9 A simple artificial neural network represented with analog genetic encoding. The interaction strengths are computed by the interaction map $I$ which takes sequences of characters of arbitrary length as arguments

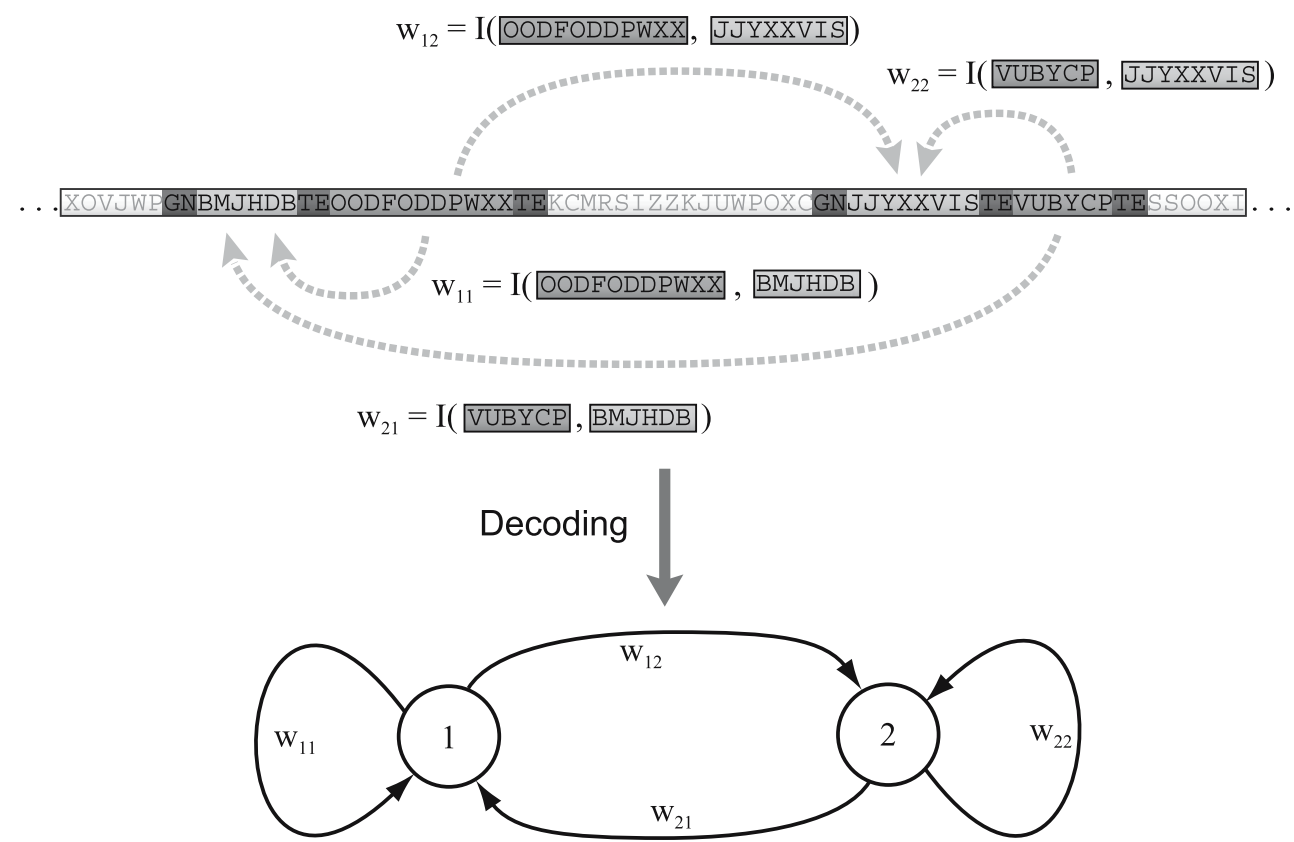

is not unduly constrained and tolerates a large class of genetic operators, which can alter both the topology and the sizing of the encoded network. In particular, the AGE genome permits the insertion, deletion and substitution of single characters, and the insertion, deletion, duplication, and transposition of whole genome fragments. All these genetic mutations are known to occur in biological genomes and to be instrumental to their evolution. In particular, gene duplication and the insertion of fragments of genome of foreign organisms are deemed to be crucial mechanism for the evolutionary increase of complexity of GRNs [75]. Finally, the interaction map is defined so as to be highly redundant, so that many different pairs of character sequences produce the same numeric value. Thus, many mutations have no effect, resulting potentially in a high neutrality in the search space.

When compared to NEAT, AGE reported equal performance on a non-Markovian double-pole balancing problem [16], while both algorithms performed better than a developmental encoding (CE) and a coevolution method (ESP). However, both AGE and NEAT performed worse than an evolution strategy with direct encoding of a fixed topology ANN [38]. This indicates that if a suitable topology is known in advance, it is better to use simpler representations. Reisinger and Miikkulainen [65] showed that an implicit encoding very similar to AGE outperforms NEAT on a complex board-game task.

\section{Neuroevolution with dynamic neuron models}

Some applications, for example the detection or generation of time sequences, require a dynamic behavior of the neural networks. Feedback connections in ANNs with static transfer functions can provide such dynamics. Another possibility is the integration of dynamic neuron models. Dynamic neural networks are more difficult to train with learning algorithms that use gradient descent, correlation, and reinforcement learning. Artificial evolution instead has been used with success on a variety of applications because it can discover suitable architectures, time constants and even learning algorithms.

\subsection{Continuous-time recurrent neural networks}

One of the most widely-used dynamic neuron models is called continuous-time recurrent neural network, or CTRNN [7]. In this model, the neural network can be seen as a set of differential equations

$$
\begin{aligned}
& \frac{d x_{i}(t)}{d t}=\frac{1}{\tau_{i}}\left(-x_{i}(t)+\sum_{j=1}^{N} w_{i j} \sigma\left(x_{j}(t)+\theta_{j}\right)+I_{i}\right) \\
& i=1,2, \ldots, N
\end{aligned}
$$

where $x_{i}(t)$ is the activation of neuron $i$ at the time $t, N$ is the number of neurons in the network, $\tau_{i}$ is a time constant whose magnitude (for $\tau_{i}>0$ ) is inversely proportional to the decay rate of the activation, $w_{i j}$ is the connection weight between neuron $i$ and neuron $j, \sigma(x)=1 /\left(1+e^{-x}\right)$ is the standard logistic activation function, $\theta_{j}$ is a bias term and $I_{i}$ represents an external input. CTRNNs display rich dynamics and represent a first approximation of the timedependent processes that occur at the membrane of biological neurons. Sometimes these models are also called leaky integrators, with reference to electrical circuits, 
because the equation describing the neuron activation is equivalent to that describing the voltage difference of a capacitor, where the time constant of the exponential and synaptic weights can be approximated by a set of resistors [31]. While being one of the simplest nonlinear, continuous dynamic network models, CTRNNs are universal dynamic approximators [24]. This means that they can approximate the trajectories of any smooth dynamical system arbitrarily well for any finite interval of time.

Evolution of CTRNNs has been applied to difficult problems such as robot control [86]. An interesting property of CTRNNs is that they can display learning behavior without synaptic plasticity because they can store intermediate states in the activation function of internal neurons. For example, Yamauchi and Beer [90] evolved CTRNNs capable of generating and learning short sequences without synaptic plasticity. Blynel and Floreano [9] evolved a recurrent neural architecture capable of reinforcement learning-like behaviors for a robot required to navigate a T-maze.

\subsection{Spiking neuron models}

Most biological neurons communicate through action potentials, or spikes, which are punctual events that result from a process taking place at the output of the neuron.

In spiking neurons the activation state, which corresponds to an analog value, can be approximated by the firing rate of the neuron. That is, a larger number of spikes within a given time window would be an indicator of higher activation of the neuron. However, if that is the case, it means that spiking neurons require relatively longer time to communicate information to post-synaptic neurons. This hints at the fact that spiking neurons may use other ways to efficiently encode information, such as the firing time of single spikes or the temporal coincidence of spikes coming from multiple sources [77, 67]. It may therefore be advantageous for engineering purposes to use models of spiking neurons that exploit firing time in order to encode spatial and temporal structure of the input patterns with less computational resources.

There are several models of spiking neurons that describe in detail the electrochemical processes that produce spiking events by means of differential equations [34]. A simple way of implementing a spiking neuron is to take the dynamic model of a CTRNN neuron and substitute the output function with an element that compares the neuron activation with its threshold followed by a pulse generator that takes the form of a Dirac function (see Fig. 10). In other words, if the neuron activation is larger than the threshold, a spike is emitted. In order to prevent continuous spike emission, one must also add a strong

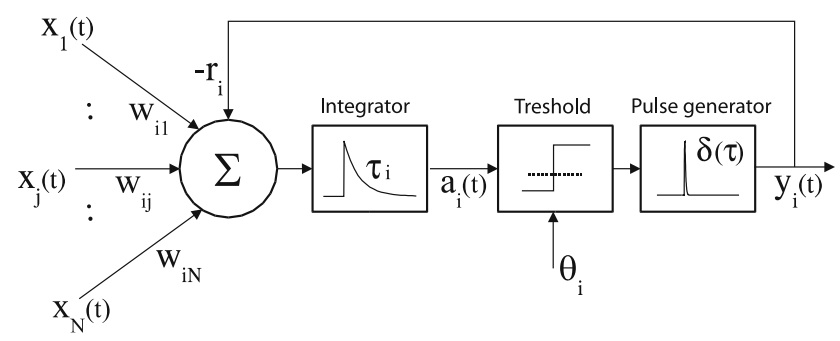

Fig. 10 The integrate and fire neuron is a simple extension of the CTRNN neuron. The weighted sum of the inputs is fed to an integrator which is followed by a threshold function and a pulse generator. If the neuron activation is larger than a threshold, a spike is emitted

negative feedback so that the neuron activation goes below threshold immediately after spike emission. This model is known as an Integrate and Fire neuron.

Korkin et al. [41] evolved spiking neural networks which could produce time-dependent waveforms, such as sinusoids. Floreano and Mattiussi [19] evolved networks of spiking neurons for solving a non-trivial vision-based navigation task. They compared a neural network using the spike response model [26] with a conventional sigmoid network which could not solve the problem. The authors suggest that the intrinsic dynamics of the spiking neural networks might provide more degrees of freedom that can be exploited by evolution to create viable controllers. Saggie et al. [70] compared spiking neural networks with conventional static ANNs in a simple setup where an agent had to search food and avoid poison in a simulated grid world. Their results indicate that for tasks with memory dependent dynamics, spiking neural networks can provide less complex solutions. Federici [17] combined an integrate and fire neuron model with a correlation-based synaptic plasticity model and a developmental encoding. His results from experiments with a simple robot navigation task indicate that such a system may allow for the efficient evolution of large networks.

\section{Evolution and learning}

One of the main advantages of using evolutionary algorithms for the design and tuning of neural networks is that evolution can be combined with learning, ${ }^{2}$ as shown in this section.

The combination of evolution and supervised learning provides a powerful synergy between complementary search algorithms [8]. For example, gradient-based learning algorithms such as back-propagation [68, 69] are sensitive to the initial weight values, which may

\footnotetext{
${ }^{2}$ Algorithms which combine evolutionary search with some kinds of local search are sometimes called memetic algorithms [53].
} 
significantly affect the quality of the trained network. In this case, evolutionary algorithms can be used to find the initial weight values of networks to be trained with backpropagation. The fitness function is computed using the residual error of the network after training with backpropagation on a given task (notice that the final weights after supervised training are not coded back into the genotype, i.e., evolution is Darwinian, not Lamarckian). Experimental results consistently indicate that networks with evolved initial weights can be trained significantly faster and better (by two orders of magnitude) than networks with random initial weights. The genetic string can also encode the values of the learning rate and of other learning parameters, such as the momentum in the case of back-propagation. In this case, Belew et al. [8] found that the best evolved networks employed learning rates ten times higher than values suggested before (i.e., much less than 1.0), but this result may depend on several factors, such as the order of presentation of the patterns, the number of learning cycles allowed before computing the fitness, and the initial weight values.

Evolutionary algorithms have been employed also to evolve learning rules. In its general form, a learning rule can be described as a function $\Phi$ of few variables, such as presynaptic activity $x_{i}$, postsynaptic activity $y_{j}$, the current value of the synaptic connection $w_{i j}$, and the learning signal $t_{j}$

$\Delta w_{i j}=\Phi\left(x_{j}, y_{i}, w_{i j}, t_{i}\right)$

Chalmers [11] suggested to describe this function as a linear combination of the products between the variables weighted by constants. These constants are encoded in a genetic string and evolved. The neural network is trained on a set of tasks using the decoded learning rule and its performance is used to compute the fitness of the corresponding learning rule. The initial synaptic weights are always set to small random values centered around zero. Chalmers employed a fitness function based on the mean square error. A neural network with a single layer of connections was trained on eight linearly separable classification tasks. The genetic algorithm evolved a learning rule similar to the delta rule by Widrow and Hoff [89]. Similar results were obtained by Fontanari and Meir [23]. Dasdan and Oflazer [14] employed a similar encoding strategy as Chalmers to evolve unsupervised learning rules for classification tasks. The authors reported that evolved rules were more powerful than comparable, human-designed rules. Baxter [6] encoded both the architecture and whether a synapse could be modified by a simple Hebb rule (the rule was predetermined). Floreano and Mondada [20] allowed evolution to choose among four Hebbian learning rules for each synaptic connection and evaluated the approach for a mobile robot requested to solve a sequential task involving multiple sensory modalities and environmental landmarks. The results indicated that the evolved robots had the ability to adapt on-the-fly to changed environmental conditions (including spatial reorganization, textures and even robot morphologies) without incremental evolution [87]. On the contrary, evolved neural networks with fixed weights could not adapt to those changed conditions. Floreano and Urzelai [22] also showed that a morphogenetic approach can greatly benefit from co-evolution of synaptic plasticity because the strengths of the growing connections are developed by learning rules that are coevolved with the developmental rules. Finally, Floreano and Urzelai [21] showed that dynamic environments favor the genetic expression of plastic connections over static connections.

DiPaolo [15] evolved spiking neural controllers modeled using the Spike Response Model and heterosynaptic variants of a learning rule called spike-timing dependent plasticity (STDP) for a robot. The learning rule was described as a polynomial expression of the STDP rule where the components of the rule were weighted by individual constants that were genetically encoded and evolved (similar to the encoding proposed by Chalmers). The author showed that evolved robots were capable of learning suitable associations between environmental stimuli and behavior.

Nolfi and Parisi [56] evolved a neural controller for a mobile robot whose output layer included two "teaching neurons" that were used to modify the connection weights from the sensory neurons to the motor neurons with backpropagation learning during the robot's lifetime. This special architecture allows for using the sensory information not only to generate behavior but also to generate teaching signals that can modify the behavior. Analysis of the evolved robots revealed that they developed two different behaviors that were adapted to the particular environment where they happened to be "born". The evolved networks did not inherit an effective control strategy, but rather a predisposition to learn to control. This predisposition to learn involved several aspects such as a tendency to move so as to experience useful learning experiences and a tendency to acquire useful adaptive characters through learning [56].

It has been known for a long time that learning may affect natural evolution [3]. Empirical evidence shows that this is the case also for artificial evolution when combined with some form of learning [55]. Hinton and Nowlan [33] proposed a simple computational model that shows how learning might facilitate and guide evolution. They considered the case where a neural network confers added reproductive fitness on an organism only if it is connected in exactly the right way. In this worst case, there is no reasonable path toward the good network and a pure 
evolutionary search can only discover which of the potential connections should be present by trying possibilities at random. In the words of the authors, the good network is "like a needle in a haystack". Hinton and Nowlan's results suggest that the addition of learning produces a smoothing of the fitness surface area around the good combination of genes (weights), which can be discovered and easily climbed by the genetic algorithm (see Fig. 11).

One of the main limitations of Hinton and Nowlan's model is that the learning space and the evolutionary space are completely correlated. ${ }^{3}$ By systematically varying the cost of learning and the correlation between the learning space and the evolutionary space, Mayley [47] showed that: (1) the adaptive advantage of learning is proportional to the correlation between the two search spaces; (2) the assimilation of characters first acquired through learning is proportional to the correlation between the two search spaces and to the cost of learning (i.e., to the fitness lost during the first part of the lifetime in which individuals have suboptimal performance); (3) in certain situations learning costs may exceed learning benefits.

In the computational literature, it is sometimes debated whether Lamarckian evolution (i.e., an evolutionary process where characters acquired through learning are directly coded back into the genotype and transmitted to offspring) could be more effective than Darwinian evolution (i.e., an evolutionary process in which characters acquired through learning are not coded back into the genotype). Ackley and Littman [1] for instance claimed that in artificial evolution, where inherited characters can easily be coded into the genotype given that the mapping between genotype and phenotype is generally quite simple, it is preferable to use Lamarckian evolution. Indeed the authors showed that Lamarckian evolution is far more effective than Darwinian evolution in a stationary environment (where the input-output mapping does not change). However, Sasaki and Tokoro [71] showed that Darwinian evolution largely outperforms Lamarckian evolution when the environment is not stationary or when different individuals are exposed to different learning experiences.

\section{Evolution of learning architectures}

Experimental data suggests that mammalian brains employ learning mechanisms that result in behaviors similar to those produced by Reinforcement learning [50, 74].

\footnotetext{
3 The two spaces are correlated if genotypes which are close in the evolutionary space correspond to phenotypes which are also close in the phenotype space.
}

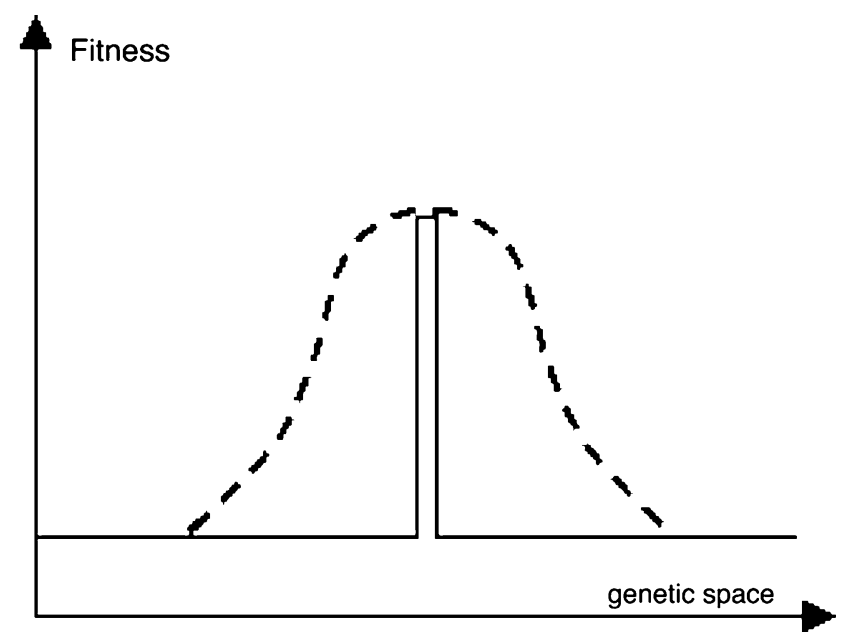

Fig. 11 Fitness landscape with and without learning. In absence of learning, the fitness landscape is flat, with a thin spike in correspondence of the good combinations of genes (thick line). When learning is enabled (dotted line), the fitness surface displays a smooth hill around the spike corresponding to the gene combination which have in part correct fixed values and in part unspecified (learnable) values. Redrawn from Hinton and Nowlan [33]

Reinforcement learning is a class of learning algorithms that attempts to estimate explicitly or implicitly the value of the states experienced by the agents in order to favor the choice of those actions that maximize the amount of positive reinforcement received by the agent over time [84]. The exact mechanism that implements this kind of learning in biological neural system is the subject of ongoing research and is not yet completely understood. The existing evidence points to the combined action of evolved value systems [58] and neuromodulatory effects [2, 18]. The value system has the task of discriminating the behaviors according to their reinforcing, punishing, or negligible consequences. This leads to the production of neuromodulatory signals that can activate or inhibit synaptic learning mechanisms.

Algorithms inspired by this approach have been developed by the machine learning community. The actor-critic model [5, 83] for example, is a simple neural architecture that implements reinforcement learning with two modules, the Actor and the Critic shown in Fig. 12. Both modules receive information on the current sensory state. In addition, the Critic receives information on the current reinforcement value (external reinforcement) from the environment. The output of the Critic generates an estimate of the weighted sum of future rewards. The output of the Actor instead is a probability of executing a certain set of actions. This allows the system to perform an exploration of the state-action space.

Typically such models are implemented in ANNs with a fixed architecture where separate modules realize the sensory preprocessing, the value system and the action- 


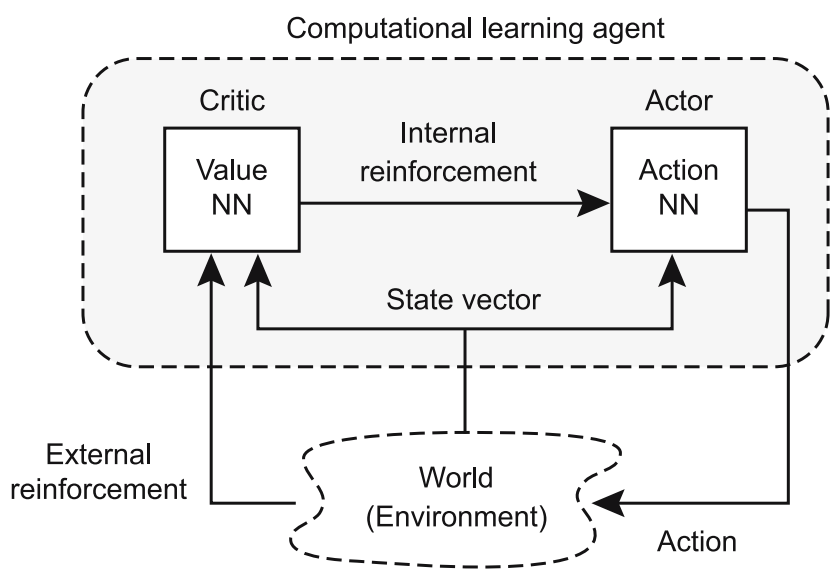

Fig. 12 The Actor-Critic architecture for reinforcement learning. Redrawn from Mizutani and Dreyfus [49]

selection mechanism. ${ }^{4}$ This puts the load of finding the optimal structure of the value and the action-selection system on the human designer. The integration of neuromodulation into a neuroevolution framework, as described in the next section, allows for the evolution of reinforcement learning-like architectures without any human intervention.

\subsection{Evolution of neuromodulatory architectures}

In biological organisms, specialized neuromodulatory neurons control the amount of activity-dependent plasticity in the connection strength of other neurons. This effect is mediated by special neurotransmitters called neuromodulators [39]. Well known neuromodulators are e.g., dopamine, serotonine, noradrenaline and acetylcholine. A simple model of neuromodulation can easily be integrated into a conventional neuroevolution framework by introducing a second type of neuron. These modulatory neurons affect the learning events at the postsynaptic site (see Fig. 13). However, the implementation of such an approach requires a genetic representation that allows for the evolution of neural architectures consisting of several different types of neurons. In Soltoggio et al. [78] this has been realized using Analog Genetic Encoding (see Sect. 2.3) and a parameterized, activity dependent Hebbian learning model [54]. The authors show how reinforcementlearning like behavior can evolve in a simulated foraging experiment. A simulated bee has to collect nectar on a field

\footnotetext{
${ }^{4}$ An alternative approach to this are neural learning classifier systems. For example, Hurst and Bull [35] addressed the control of a simulated robot in a maze task. They used a population of neural networks acting as 'rules' controlling the robot. As evolution favored rules that led to succesful behavior, the set of rules adapted to the requirements of the task.
}

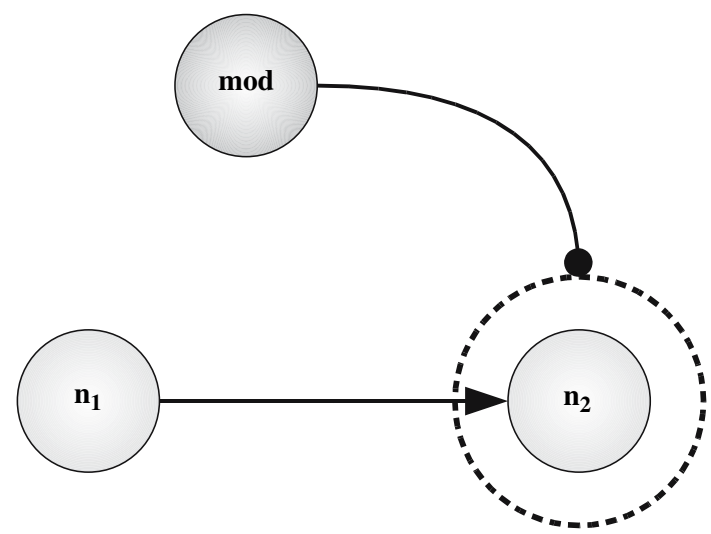

Fig. 13 Neuromodulatory neurons permit the implementation of a value-based learning mechanism in artificial neural networks. The basic learning mechanism changes the weight of the link between the two neurons $n_{1}$ and $n_{2}$ according to their activity. The neuromodulatory neuron $\bmod$ modulates (i.e., strengthens or suppresses) the basic learning mechanism and permits the synthesis of networks where the learning is activated only in particular circumstances. From Mattiussi et al. [46]

that contains two types of flowers (see Fig. 14). As the amount of nectar associated with each of the flowers changes stochastically, the behavioral strategy to maximize the amount of collected nectar has to be adapted. The evolved networks (see Fig. 15) implemented a value-based learning strategy which was found to generalize to scenarios different from those used to assess the quality of the solution during evolution, outperforming the results obtained with hand-designed value-based learning architectures [78].

Another approach to evolving neuromodulatory architectures was suggested by Husbands et al. [37]. They developed and evolved a class of neural networks called GasNets, inspired by the modulatory effects of freely diffusing gases, such as nitric oxide, which affect the response profile of neurons in biological neural networks. In GasNets, some of the neurons, which are spatially distributed over a 2D surface, emit 'gases' that diffuse through the network and modulate the profile of the activation function of other neurons in their vicinity. The spatial distance between the neurons determines both the gas concentration (as a result of the diffusion dynamics) and, in combination with additional genetic parameters, the network connectivity.

The authors showed that the modulation caused by gas diffusion introduces a form of plasticity in the network without synaptic modification. A comparison with conventional neural networks in a robot control task indicated better evolvability of the GasNets [37]. In another set of experiments, McHale and Husbands [48] compared GasNets to CTRNNs and conventional ANNs with Hebbian learning in a robot locomotion control tasks. From all 


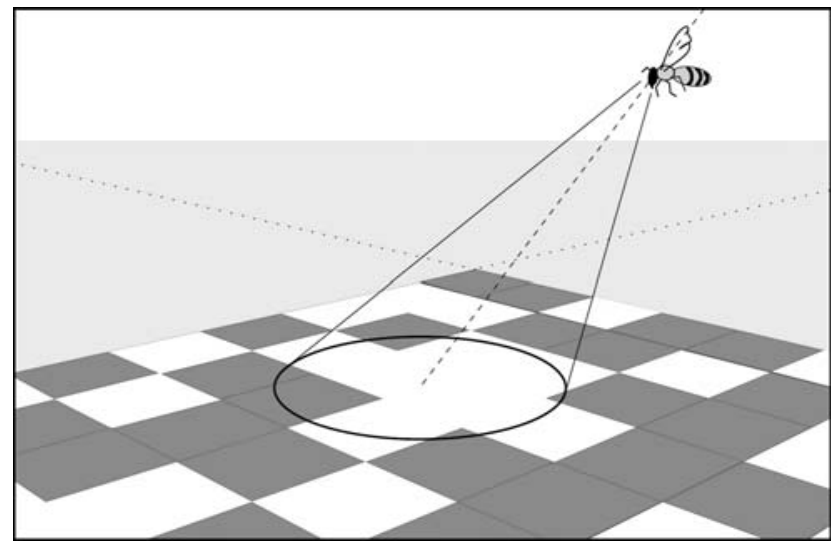

Fig. 14 A simulated bee with a simple vision system flies over a field containing patches of blue and yellow flowers, represented here as dark and light squares. The quality of the behavior of the simulated bee is judged from the amount of nectar that the bee is able to collect in a series of landings. From Soltoggio et al. [78]

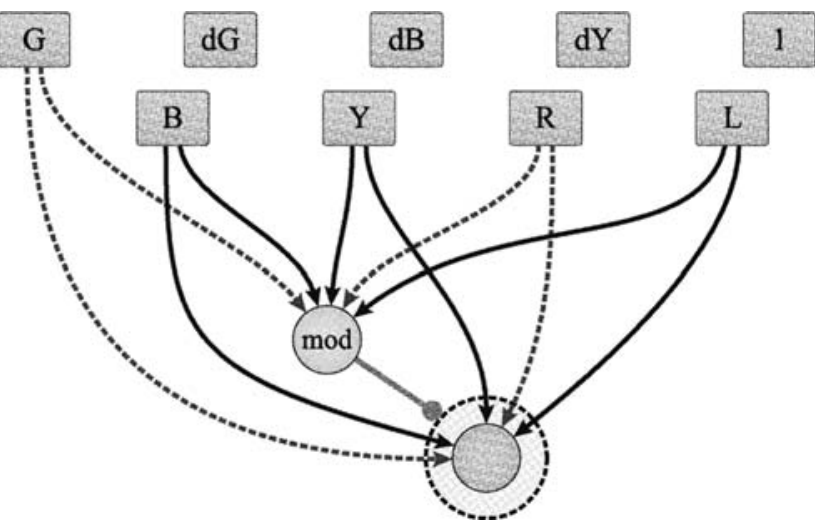

Fig. 15 An example of a neuromodulatory network evolved with analog genetic encoding, which solves the foraging task of Fig. 14. The $G, B$, and $Y$ squares represent the color inputs of the visual system. The $R$ square represents the reward input that gives the amount of nectar found on the flower on which the simulated bee has landed. The landing is signaled by the activation of the input $\mathrm{L}$. The square denoted by 1 represents a fixed value input (bias), and the $\mathrm{dG}$, $\mathrm{dB}, \mathrm{dY}$ inputs represent the memory of the color observed just before landing. The $\mathrm{dG}, \mathrm{dB}$, and $\mathrm{dY}$ input signals were defined because they were used in experiments with hand-designed neural architectures for the same scenario [54]. The figure shows that these inputs were found by the algorithm to be not necessary to solve the task and were thus left unconnected at the end of the evolutionary search. In the network shown here, the neuromodulatory mod neuron modulates the learning of all the connections between the inputs and the output neuron. From Soltoggio et al. [78]

tested networks, only the GasNets achieved a cyclic locomotion, but the CTRNNs achieved a higher average fitness. This is in line with Magg and Philippides [43] whose results indicate that GasNets perform extremely well on tasks that require some kind of neural pattern generator or timer, while performing worse on tasks which do not require different time scales in the network.

\section{Closing remarks}

The methods for evolving neural networks have greatly advanced over the years. In particular, we have witnessed a significant progress in the ability to evolve architectures and learning for dynamic neural networks, which are very powerful systems for real-world problems such as robot control.

From a practical point of view, artificial evolution turns out to be extremely efficient, or even unique, when it is used to explore both the topology and the adaptive properties of neural networks because other training algorithms usually require stronger assumptions and constraints on the choice of architecture, training data distribution, and optimization function. Although there are no rigorous comparisons between alternative methods, this claim is supported by the fact that most experiments reported in the literature with neural robots in real environments resort to some sort of evolutionary procedure rather than to other types of training algorithms.

From a scientific point of view, artificial evolution can be used to test hypothesis of brain development and dynamics because it can encompass multiple temporal and spatial scales along which an organism evolves, such as genetic, developmental, learning, and behavioral phenomena. The possibility to co-evolve both the neural system and the morphological properties of agents (although this latter aspect is not reviewed in this paper) adds an additional valuable perspective to the evolutionary approach that cannot be matched by any other approach.

We think that neuroevolution will continue to provide efficient solutions to hard problems as well as new insights into how biological brains became what they are in physical bodies interacting with dynamic environments. Within this context, we believe that the evolution of neural architectures with elements that gate or modulate learning will generate novel understanding of reinforcement-learning like structures that are so important for biological and robotic life.

Acknowledgements This work was supported by the Swiss National Science Foundation, grant no. 200021-112060. Thanks to Daniel Marbach for the illustrations and the two anonymous reviewers for their helpful comments.

\section{References}

1. Ackley DH, Littman ML (1992) Interactions between learning and evolution. In: Langton C, Farmer J, Rasmussen S, Taylor C (eds) Artificial Life II: Proceedings volume of Santa Fe conference, vol XI. Addison Wesley, Redwood City, pp 487-510

2. Bailey CH, Giustetto M, Huang Y.-Y, Hawkins RD, Kandel ER (2000) Is heterosynaptic modulation essential for stabilizing Hebbian plasticity and memory? Nat Rev Neurosci 1(1):11-20 
3. Baldwin JM (1896) A new factor in evolution. Am Nat 30:441451

4. Banzhaf W, Nordin P, Keller RE, Francone FD (1998) Genetic programming - an introduction. In: On the automatic evolution of computer programs and its applications. Morgan Kaufmann, San Francisco

5. Barto AG (1995) Adaptive critic and the basal ganglia. In: Houk JC, Davis JL, Beiser DG (eds) Models of information processing in the basal ganglia. MIT Press, Cambridge, pp 215-232

6. Baxter J (1992) The evolution of learning algorithms for artificial neural networks. In: Green D, Bossomaier T (eds) Complex Systems. IOS Press

7. Beer RD, Gallagher JC (1992) Evolving dynamical neural networks for adaptive behavior. Adapt Behav 1:91-122

8. Belew RK, McInerney J, Schraudolph NN (1992) Evolving networks: using the genetic algorithm with connectionistic learning. In: Langton CG, Taylor C, Farmer JD, Rasmussen S (eds) Proceedings of the 2nd Conference on Artificial Life. AddisonWesley, Reading, pp 511-548

9. Blynel J, Floreano D (2003) Exploring the T-maze: evolving learning-like robot behaviors using CTRNNs. In: Raidl Ge AE (ed) 2nd European workshop on evolutionary robotics (EvoRob'2003)

10. Bongard J (2002) Evolving modular genetic regulatory networks. In: Proceedings of the 2002 congress on evolutionary computation 2002, CEC '02, vol 2, pp 1872-1877

11. Chalmers DJ (1990) The evolution of learning: an experiment in genetic connectionism. In: Touretzky DS, Elman JL, Sejnowski T, Hinton GE (eds) Proceedings of the 1990 connectionist models summer school. Morgan Kaufmann, San Mateo, pp 81-90

12. Chandra A, Yao X (2006) Ensemble learning using multiobjective evolutionary algorithms. J Math Model Algorithms 5(4):417-445

13. Chellapilla K, Fogel D (2001) Evolving an expert checkers playing program without using humanexpertise. IEEE Trans Evol Comput 5(4):422-428

14. Dasdan A, Oflazer K (1993) Genetic synthesis of unsupervised learning algorithms. In: Proceedings of the 2nd Turkish symposium on artificial intelligence and ANNs. Department of Computer Engineering and Information Science, Bilkent University, Ankara

15. DiPaolo E (2003) Evolving spike-timing-dependent plasticity for single-trial learning in robots. Phil Trans R Soc Lond A 361:2299-2319

16. Dürr P, Mattiussi C, Floreano D (2006) Neuroevolution with Analog Genetic Encoding. In: Parallel problem solving from nature-PPSN iX, vol 9. Springer, Berlin, pp 671-680

17. Federici D (2005) Evolving developing spiking neural networks. In: Proceedings of CEC 2005 IEEE congress on evolutionary computation

18. Fellous J-M, Linster C (1998) Computational models of neuromodulation. Neural Comput 10(4):771-805

19. Floreano D, Mattiussi C (2001) Evolution of spiking neural controllers for autonomous vision-based robots. In: Gomi T (ed) Evolutionary robotics. From intelligent robotics to artificial life. Springer, Tokyo

20. Floreano D, Mondada F (1996) Evolution of plastic neurocontrollers for situated agents. In: Maes P, Matarić M, Meyer J, Pollack J, Roitblat H, Wilson S (eds) From animals to animats IV: proceedings of the 4 th international conference on simulation of adaptive behavior. MIT Press-Bradford Books, Cambridge, pp 402-410

21. Floreano D, Urzelai J (2000) Evolutionary robots with online self-organization and behavioral fitness. Neural Netw 13:431-443

22. Floreano D, Urzelai J (2001) Evolution of plastic control networks. Autonom Robots 11(3):311-317
23. Fontanari JF, Meir R (1991) Evolving a learning algorithm for the binary perceptron. Network 2:353-359

24. Funahashi K, Nakamura Y (1993) Approximation of dynamical systems by continuous time recurrent neural networks. Neural Netw 6(6):801-806

25. Geard NL, Wiles J (2003) Structure and dynamics of a gene network model incorporating small RNAs. In: Proceedings of 2003 congress on evolutionary computation, pp 199-206

26. Gerstner W (1999) Spiking neurons. In: Maass W, Bishop CM (eds) Pulsed neural networks. MIT Press-Bradford Books, Cambridge

27. Gomez F, Miikkulainen R (1997) Incremental evolution of complex general behavior. Adapt Behav 5(3-4):317-342

28. Gruau F (1995) Automatic definition of modular neural networks. Adapt Behav 3(2):151-183

29. Gruau, F, Whitley, D, and Pyeatt, L (1996) A comparison between cellular encoding and direct encoding for genetic neural networks. In: Koza JR, Goldberg DE, Fogel DB, Riolo RL (eds) Genetic programming 1996: proceedings of the first annual conference. MIT Press, Stanford University, pp 81-89

30. Hansen N, Ostermeier A (2001) Completely derandomized selfadaptation in evolution strategies. Evol Comput 9(2):159-195

31. Haykin, S (1999) Neural networks. a comprehensive foundation, 2nd edn. Prentice Hall, Upper Saddle River

32. Hebb DO (1949) The organisation of behavior. Wiley, New York

33. Hinton GE, Nowlan SJ (1987) How learning can guide evolution. Complex Syst 1:495-502

34. Hodgkin AL, Huxley AF (1952) A quantitative description of membrane current and its application to conduction and excitation in nerve. J Physiol (Lond) 108:500-544

35. Hurst J, Bull L (2006) A neural learning classifier system with self-adaptive constructivism for mobile robot control. Artif Life $12(3): 353-380$

36. Husbands P, Harvey I, Cliff D, Miller G (1994) The use of genetic algorithms for the development of sensorimotor control systems. In: Gaussier P, Nicoud J-D (eds) From perceptin to action. IEEE Press, Los Alamitos

37. Husbands P, Smith T, Jakobi N, O'Shea M (1998) Better living through chemistry: evolving gasnets for robot control. Connect Sci 10:185-210

38. Igel, C (2003) Neuroevolution for reinforcement learning using evolution strategies. In: Sarker R, et al (eds) Congress on evolutionary computation, vol 4. IEEE Press, New York, pp 2588 2595

39. Katz PS (1999) What are we talking about? Modes of neuronal communication. In: Katz $\mathrm{P}$ (eds) Beyond neurotransmission: neuromodulation and its importance for information processing, chap 1. Oxford University Press, Oxford, pp 1-28

40. Kitano H (1990) Designing neural networks by genetic algorithms using graph generation system. Complex Syst J 4:461476

41. Korkin M, Nawa NE, de Garis H (1998) A 'spike interval information coding' representation for ATR's CAM-brain machine (CBM) In: Proceedings of the 2nd international conference on evolvable systems: from biology to hardware (ICES'98). Springer, Heidelberg

42. Koza JR (1994) Genetic programming II: automatic discovery of reusable programs. MIT Press, Cambridge

43. Magg S, Philippides A (2006) Gasnets and CTRNNs : a comparison in terms of evolvability. In: From animals to animats 9: proceedings of the 9th international conference on simulation of adaptive behavior. Springer, Heidelberg, pp 461-472

44. Mattiussi C, Floreano D (2004) Evolution of analog networks using local string alignment on highly reorganizable genomes. In: Zebulum RS et al (eds) NASA/DoD conference on evolvable hardware (EH'2004), pp 30-37 
45. Mattiussi C, Dürr P, Floreano D (2007a) Center of mass encoding: a self-adaptive representation with adjustable redundancy for real-valued parameters. In: GECCO 2007. ACM Press, New York, pp 1304-1311

46. Mattiussi C, Marbach D, Dürr P, Floreano D (2007b) The age of analog networks. AI Magazine (in press)

47. Mayley G (1996) Landscapes, learning costs and genetic assimilation. Evol Comput 4(3):213-234

48. McHale G, Husbands P (2004) Gasnets and other evolvable neural networks applied to bipedal locomotion. In: Schaal S (ed) Proceedings from animals to animats 8: proceedings of the 8th international conference on simulation of adaptive behaviour (SAB'2004). MIT Press, Cambridge, pp 163-172

49. Mizutani E, Dreyfus SE (1998) Totally model-free reinforcement learning by actor-critic elman networks in non-markovian domains. In: Proceedings of the IEEE world congress on computational intelligence. IEEE Press, New York

50. Montague P, Dayan P, Sejnowski T (1996) A framework for mesencephalic dopamine systems based on predictive Hebbian learning. J Neurosci 16(5):1936-1947

51. Montana D, Davis L (1989) Training feed forward neural networks using genetic algorithms. In: Proceedings of the 11th international joint conference on artificial intelligence. Morgan Kaufmann, San Mateo, pp 529-538

52. Moriarty DE, Miikkulainen R (1996) Efficient reinforcement learning through symbiotic evolution. Machine Learn 22:11-32

53. Moscato P (1989) On evolution, search, optimization, genetic algorithms and martial arts: towards memetic algorithms. In: Technical report C3P 826, Pasadena

54. Niv Y, Joel D, Meilijson I, Ruppin E (2002) Evolution of reinforcement learning in uncertain environments: A simple explanation for complex foraging behaviors. Adapt Behav 10(1):5-24

55. Nolfi S, Floreano D (1999) Learning and evolution. Auton Robots 7(1):89-113

56. Nolfi S, Parisi D (1996) Learning to adapt to changing environments in evolving neural networks. Adapt Behav 5(1):75-98

57. Nolfi S, Miglino O, Parisi D (1994) Phenotypic plasticity in evolving neural networks. In: Gaussier P, Nicoud J-D (eds) From perception to action. IEEE Press, Los Alamitos

58. Pfeifer R, Scheier C (1999) Understanding Intelligence. MIT Press, Cambridge

59. Purves D (1994) Neural activity in the growth of the brain. Cambridge University Press, Cambridge

60. Quartz S, Sejnowski TJ (1997) The neural basis of cognitive development: a constructivist manifesto. Behav Brain Sci 4:537555

61. Radcliffe NJ (1991) Form an analysis and random respectful recombination. In: Belew RK, Booker LB (eds) Proceedings of the 4th international conference on genetic algorithms. Morgan Kaufmann, San Mateo

62. Rechenberg I (1973) Evolutionsstrategie-Optimierung technischer Systeme nach Prinzipien der biologischen Evolution. Fommann-Holzboog, Stuttgart

63. Reil T (1999) Dynamics of gene expression in an artificial genome-implications for biological and artificial ontogeny. In: Proceedings of the 5th European conference on artificial life, pp 457-466

64. Reil T (2003) On growth, form and computers. In: Artificial genomes as models of gene regulation. Academic Press, London, pp 256-277

65. Reisinger J, Miikkulainen R (2007) Acquiring evolvability through adaptive representations. In: Proceedings of genetic and evolutionary computation conference (GECCO 2007)

66. Reisinger J, Bahçeci E, Karpov I, Miikkulainen R (2007) Coevolving strategies for general game playing. In: Proceedings of the IEEE symposium on computational intelligence and games (CIG-2007)

67. Rieke F, Warland D, van Steveninck R, Bialek W (1997) Spikes. Exploring the neural code. MIT Press, Cambridge

68. Rumelhart DE, Hinton GE, Williams RJ (1986a) Learning representations by back-propagation of errors. Nature 323:533-536

69. Rumelhart DE, McClelland J, the PDP Research Group (1986b) Parallel distributed processing: explorations in the microstructure of cognition. Foundations, vol 1. MIT Press-Bradford Books, Cambridge

70. Saggie K, Keinan A, Ruppin E (2004) Spikes that count: rethinking spikiness in neurally embedded systems. Neurocomputing 58-60:303-311

71. Sasaki T, Tokoro M (1997) Adaptation toward changing environments: Why Darwinian in nature? In: Husbands P, Harvey I (eds) Proceedings of the 4th European conference on artificial life. MIT Press, Cambridge

72. Schaffer JD, Whitley D, Eshelman LJ (1992) Combinations of genetic algorithms and neural networks: a survey of the state of the art. In: Whitley D, Schaffer JD (eds) Proceedings of an international workshop on the combinations of genetic algorithms and neural networks (COGANN-92). IEEE Press, New York

73. Schraudolph NN, Belew RK (1992) Dynamic parameter encoding for genetic algorithms. Machine Learn 9:9-21

74. Schultz W, Dayan P, Montague PR (1997) A neural substrate of prediction and reward. Science 275(5306):1593-1599

75. Shapiro J (2005) A 21st century view of evolution: genome system architecture, repetitive DNA, and natural genetic engineering. Gene 345(1):91-100

76. Siddiqi A, Lucas S (1998) A comparison of matrix rewriting versus direct encoding for evolving neural networks. In: Proceedings of the 1998 IEEE international conference on evolutionary computation. Piscataway, NJ, pp 392-397

77. Singer W, Gray CM (1995) Visual feature integration and the temporal correlation hypothesis. Annu Rev Neurosci 18:555586

78. Soltoggio A, Duerr P, Mattiussi C, Floreano D (2007) Evolving neuromodulatory topologies for reinforcement learning-like problems. In: Angeline P, Michaelewicz M, Schonauer G, Yao X, Zalzala Z (eds) Proceedings of the 2007 congress on evolutionary computation. IEEE Press, New York

79. Stanley K, Miikkulainen R (2002) Evolving neural networks through augmenting topologies. Evol Comput 10(2):99-127

80. Stanley KO, Miikkulainen R (2004) Competitive coevolution through evolutionary complexification. J Artif Intell Res 21:63100

81. Stanley K, Kohl N, Sherony R, Miikkulainen R (2005a) Neuroevolution of an automobile crash warning system. In: Proceedings of genetic and evolutionary computation conference (GECCO 2005)

82. Stanley KO, Cornelius R, Miikkulainen R, D’Silva T, Gold A (2005b) Real-time learning in the nero video game. In: Proceedings of the artificial intelligence and interactive digital entertainment conference (AIIDE 2005) demo papers

83. Sutton RS (1988) Learning to predict by the method of temporal difference. Machine Learn 3:9-44

84. Sutton RS, Barto AG (1998) Reinforcement learning. an introduction. MIT Press, Cambridge

85. Trianni V, Ampatzis C, Christensen A, Tuci E, Dorigo M, Nolfi S (2007) From solitary to collective behaviours: decision making and cooperation. In: Advances in artificial life, proceedings of ECAL 2007. Lecture Notes in Artificial Intelligence, vol LNAI 4648. Springer, Berlin, pp 575-584

86. Tuci E, Quinn M, Harvey I (2002) An evolutionary ecological approach to the study of learning behavior using a robot-based model. Adapt Behav 10(3-4):201-221 
87. Urzelai J, Floreano D (2001) Evolution of adaptive synapses: robots with fast adaptive behavior in new environments. Evol Comput 9:495-524

88. Whitley D, Starkweather T, Bogart C (1990) Genetic algorithms and neural networks: optimizing connections and connectivity. Parallel Comput 14:347-361

89. Widrow B, Hoff ME (1960) Adaptive switching circuits. In: Proceedings of the 1960 IRE WESCON convention, vol IV, New
York. IRE. Reprinted in Anderson and Rosenfeld, 1988, pp 96104

90. Yamauchi BM, Beer RD (1994) Sequential behavior and learning in evolved dynamical neural networks. Adapt Behav 2(3):219246

91. Yao X (1999) Evolving artificial neural networks. Proc IEEE 87(9):1423-1447 